\title{
Estimating codend size selectivity of bottom trawlnet in Chilean hake (Merluccius gayi gayi) fishery
}

\author{
Mauricio Gálvez ${ }^{1}$ \& Hernán Rebolledo ${ }^{2}$ \\ ${ }^{1}$ Subsecretaría de Pesca, Bellavista 168, Piso 14, Casilla 100-V, Valparaíso, Chile \\ ${ }^{2}$ Instituto de Investigación Pesquera, Av. Cristóbal Colón 2780, Casilla 350, Talcahuano, Chile
}

\begin{abstract}
This paper examines codend size selectivity in the Chilean hake (Merluccius gayi gayi) industrial fishery by means of four mesh sizes opening in the codend (100, 110, 130 and $140 \mathrm{~mm}$ mesh openings) and using the cover codend method. The data was analyzed by haul, using the SELECT model with both the Logistic and Richards selection curves. For each mesh size opening and sex the combined haul analysis was made with the Fryer model, and with these results we determine the Master Curves, by sex. Based on critical length and maturity length at 50\% estimated for this specie we determined the optimum mesh size opening for the trawl fishery. The rate of escape of cmomon hake increased exponentially with the size of mesh opening. The Logistic selection model was chosen based on the likelihood ratio test for all hauls analyzed. From the mean selection curves (incorporating haul variation) fitted to data for combined sexes, the total length of $50 \%$ retention $\left(l_{50}\right)$ were estimated to be $39.3,39.7,41.1$ and $43.6 \mathrm{~cm}$ of total length for 100 , 110,130 and $140 \mathrm{~mm}$ of mesh size opening. The values of $l_{50}$ for females were higher than those for males. We recommended $120 \mathrm{~mm}$ of mesh size opening in the codend in order to protect the spawning fraction of Chilean hake stock and maximize the biomass of the cohorts.
\end{abstract}

Key words: size selectivity, bottom trawl net, codend method, SELECT model, Master Curve, hake Merluccius gayi gayi, Chile.

\section{Estimación de la selectividad a la talla por copos de redes de arrastre de fondo en la pesquería de merluza común (Merluccius gayi gayi)}

RESUMEN. El presente artículo examina la selectividad a la talla en la pesquería de arrastre de merluza común (Merluccius gayi gayi), mediante el empleo de cuatro tamaños de luz de malla en el copo (100, 110, 130 y $140 \mathrm{~mm}$ ) y el uso de un cubre-copo. Los datos fueron analizados por lance, mediante el procedimiento SELECT, utilizando los modelos de selección Logita y de Richard. Se realizó un análisis combinado de los lances de pesca, por tamaño de luz de malla y sexo, a través del modelo de Fryer, y con estos resultados se determinó la Curva Maestra, por sexo. Basados en estimaciones previas de la talla crítica y la talla de madurez sexual al 50\%, se determinó el tamaño de luz de malla óptimo para la pesquería de arrastre. Los resultados indicaron que la tasa de escape de merluza se incrementa exponencialmente con el aumento del tamaño de luz de malla en el copo. A través del test de razón de verosimilitud, se determinó que el modelo de selección que mejor se ajusta a los datos es Logita. A partir de las curvas medias de selección (incorporando la variación entre lances) sin diferenciación de sexo, se determinaron las longitudes de retención al 50\% $\left(l_{50}\right)$ de 39,3; 39,7; 41,1 y 43,6 cm para los tamaños de luz de malla de 100, 110, 130 y $140 \mathrm{~mm}$, respectivamente. Las estimaciones de $l_{50}$ en las hembras fueron un poco más altas que en machos. Se recomienda un tamaño de luz de malla de $120 \mathrm{~mm}$ en los copos, en orden a proteger la fracción desovante de merluza común y maximizar la biomasa de las cohortes.

Palabras clave: selección a la talla, red de arrastre, método de cubre-copo, modelo SELECT, Curva Maestra, Merluccius gayi gayi, Chile. 


\section{INTRODUCTION}

Since the 1940s, Chilean hake (Merluccius gayi gayi) has been one of the most important demersal fishing resources for both artisanal and industrial sectors in Chile. At present, the stock of Chilean hake is full exploited and the fishery is managed by setting an annual quota (73,000 ton in year 2005), and also by setting a Minimum Legal Mesh Size (MLMS) to minimize the impact on juveniles. A Total Allowable Catch (TAC) is yearly estimated and hydroacustic survey is carried out almost every years. However, the size-selection scheme has not been revised for 24 years, when the size of mesh opening in the codend was fixed at $100 \mathrm{~mm}$ (Supreme Decree No. 238 of 1982).

In recent years fishing power of industrial fleet has significantly increased with new fish finder on board, large and more efficient fishing gears, changes in net design, increasing towing power and hold capacity of the vessels. The annual quota has increased from 45,000 metric ton per year during 1983-1991 period to 139,500 metric tons in year 2004, recruitment has strongly varied, and two strong El Niño events have occurred (1982-83 and 1997-98). In year 2004 the catch of trawler fleet have been heavy centered on juvenile hakes, with a strong biomass decrease of the spawning stock (SUBPESCA, 2004). All these changes suggest a review of the size-selectivity of Chilean hake, in order to recover the stock biomass, to maintain the sustainability of the fishery and to maximize the yield per recruit.

Furthermore, previous studies on Chilean hake selectivity (Saetersdal \& Villegas, 1968; Arana, 1970; Klenner, 1978; Pavez, 1981) were not carried out with rigorous statistical analysis of the data. In fact, those studies did not use adequate analytical approaches for combined haul analysis, and the variation between hauls was ignored.

In this study we established the size selection curves by sex for the current MLMS in the codend, and also for three additional mesh sizes opening $(110,130$ and $140 \mathrm{~mm})$. For this purpose, maximum likelihood procedures (SELECT model; Millar, 1991) for haul-to-haul analysis was used, and the between-haul variation model of Fryer (1991) was applied to provide standard errors of the parameters. The Master Curve with the Tokai \& Kitahara (1989) method was used to determine the retention probability for any mesh size opening between 100 and $140 \mathrm{~mm}$, and for any fish length between the observed lengths. Finally, we consider length of 50\% of maturity $\left(\mathrm{L}_{\mathrm{m}}\right.$; Froese \& Pauly, 1998) and the age ( $\mathrm{T}_{\mathrm{mb}}$, converted to length) at which the cohort maximizes its biomass (Alverson \& Carney, 1975), to determine the mesh size opening that generate a length of $50 \%$ of retention $\left(l_{50}\right)$ equal or higher than $\mathrm{L}_{\mathrm{m}}$ and $\mathrm{T}_{\mathrm{mb}}$.

\section{MATERIALS AND METHODS}

\section{Experimental design and data}

Total length frequency data by sex of $M$. gayi gayi was obtained from 32 covered codend experimental hauls (Fig. 1). The experiments were carried out with codends of $100 \mathrm{~mm}$ (9 hauls), $110 \mathrm{~mm}$ ( 8 hauls), $130 \mathrm{~mm}$ (7 hauls) and $140 \mathrm{~mm}$ (8 hauls) mesh openings. The experimental catches were conducted by using a stern trawl commercial fishing vessel «Biomar III» (41.71 m length; $1900 \mathrm{HP}$ ) during March-April 2000, in the central-southern area of Chile (between $34^{\circ} 50^{\prime} \mathrm{S}, 72^{\circ} 20^{\prime} \mathrm{W}$ and $35^{\circ} 40^{\prime} \mathrm{S}$, $\left.73^{\circ} 10^{\prime} \mathrm{W}\right)$. All experimental hauls were carried out during the day at average speed of 3.4 knots and tow duration of 30 to 50 minutes. The depth in fishing ground ranged from 90 to $280 \mathrm{~m}$.

The net used in the experimental catches was an «Engel Balloon Trawl» type with a headline of 53 $\mathrm{m}$ and a groundrope line of $37 \mathrm{~m}$. Four types of detachable codends with diamond-shaped mesh that had different mesh size and twine construction were used in the experiments (Table 1). In order to retain fishes that escaped from the codend, a cover net was installed around the extension piece and codend. The cover net mesh (control codend) was diamondshaped and had $60 \mathrm{~mm}$ mesh opening in the upper panel. The lower panel of the cover net was constructed with polyamide (PA, $49 \mathrm{~mm}$ mesh opening) in order to keep contact with the bottom and does not obstruct the escape of fish from the lower panel of the codend. The upper panel of the cover net was constructed with polyethylene (PE) and with added uniform buoyancy, so that the upper panel of the cover net did not remain in contact with the upper panel of the codend. All meshes were measure with a metallic device with isosceles triangle shape and graduated each $2 \mathrm{~mm}$.

\section{Individual hauls analysis}

The data obtained by haul was analyzed with the SELECT model of Millar (1991) and Millar \& Walsh 


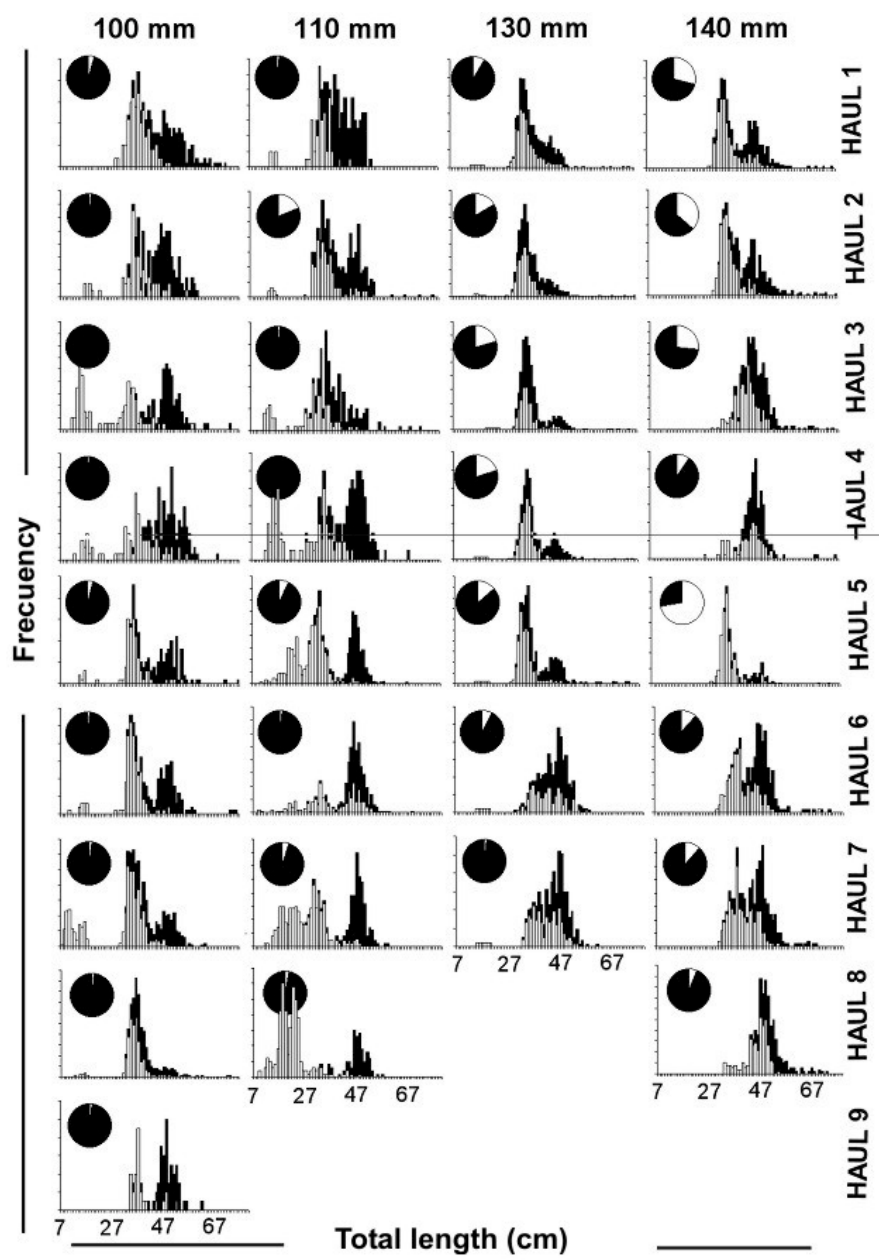

Figure 1. Chilean hake (female + male + undetermined) total length distribution in the codend (fill bar) and cover (empty bar), by size of mesh opening and haul. The proportion (in weight) of catch retained (filled) and escaped (empty) from the experimental codend is given in the circle.

Figura 1. Distribución de frecuencias de longitud total de merluza común (hembras + machos + indeterminados) en el copo (barra negra) y en el cubre-copo (barra blanca), según tamaño de luz de malla y lance. El círculo muestra la proporción (en peso) de la captura retenida (en negro) y escapada (en blanco) del copo experimental.

Table 1. Codend and cover net specifications. SD: Standard deviation.

Tabla 1. Especificaciones del copo y cubre-copo. SD: Deviación estándar.

\begin{tabular}{|c|c|c|c|c|c|c|c|c|}
\hline & \multirow[b]{2}{*}{$\begin{array}{l}\text { Number } \\
\text { measured }\end{array}$} & \multicolumn{2}{|l|}{ Mesh opening } & \multicolumn{2}{|c|}{ Dimension } & \multirow[b]{2}{*}{ Material } & \multicolumn{2}{|c|}{ Twine } \\
\hline & & $\begin{array}{l}\text { Average size } \\
\quad(\mathbf{m m})\end{array}$ & SD & $\begin{array}{l}\text { Length } \\
\text { (m) }\end{array}$ & $\begin{array}{l}\text { Wide } \\
\text { (mesh) }\end{array}$ & & $\begin{array}{l}\text { Cons- } \\
\text { truction }\end{array}$ & $\begin{array}{c}\text { Diameter } \\
(\mathbf{m m})\end{array}$ \\
\hline Codend 1 & 100 & $100.4^{3} 100$ & 1.5 & 20 & 100 & PE & Braided & $\mathrm{d} 4$ \\
\hline Codend 2 & 100 & $109.8^{3} 110$ & 1.8 & 20 & 91 & PE & Braided & $\mathrm{d} 4$ \\
\hline Codend 3 & 100 & $129.7^{3} 130$ & 2.9 & 20 & 78 & $\mathrm{PE}$ & Twisted & 8 \\
\hline Codend 4 & 100 & $140.3^{3} 140$ & 1.3 & 20 & 72 & PE & Twisted & 8 \\
\hline Cover & & & & & & & & \\
\hline (Upper panel) & 50 & 60.4 & 1.2 & 19 & 215 & PE & Twisted & 6 \\
\hline (Lower panel) & 50 & 48.9 & 0.9 & 19 & 271 & $\mathrm{PA}$ & Twisted & 7 \\
\hline
\end{tabular}


(1992), extended by Wileman et al. (1996) to the size frequency data obtained from cover-codend method. In this model, let $\mathrm{n}_{l 1}$ and $\mathrm{n}_{l 2}$ the number of length $l$ fish (in this case total length) that are taken in the codend and cover respectively, and let $\mathrm{n}_{l_{+}}=$ $\mathrm{n}_{l 1}+\mathrm{n}_{l 2}$ denote the total catch of length $l$ fish. The probability of a length $l$ fish being retained in the codend is the selection probability $\mathrm{r}(l)$. Then, assuming $\mathrm{n}_{l 1}$ to be binomially distributed with parameters $\mathrm{n}_{l_{+}}$and $\mathrm{r}(l)$, the log-likelihood function to be maximized for the data is

$$
\sum_{l}\left(n_{l 1} \log _{e} r(l)+n_{l 2} \log _{e}(1-r(l))\right)
$$

over all possible values of $\mathrm{r}(l)$ parameters.

To model the size selection, $\mathrm{r}(l)$, two types of selection curves were used. The first and more commonly selection curve is the Logistic curve:

$$
r(l)=\frac{\exp (a+b l)}{1+\exp (a+b l)}
$$

and the last one is Richards selection curve, which includes an asymmetry parameter $(\delta)$ in the form

$$
r(l)=\left(\frac{\exp (a+b l)}{1+\exp (a+b l)}\right)^{1 / \delta}
$$

The estimation process of the variance of parameters, lengths of $\mathrm{x} \%$ of retention $\left(l_{\mathrm{x}}\right)$, selection range (SR) and its variance is described in detail by Wileman et al. (1996).

The model deviance was used for hypothesis testing between Richards and Logistic selection curves. The model deviance was calculated as

$$
D=\sum_{l} D_{l}^{2}
$$

where

$D_{l}=\operatorname{sign}\left(y_{l}-\hat{y}_{l}\right) \cdot$

$\left\{2 n_{l}+\left[y_{l} \log _{e}\left(\frac{y_{l}}{\hat{y}_{l}}\right)+\left(1-y_{l}\right) \log _{e}\left(\frac{1-y_{l}}{1-\hat{y}_{l}}\right)\right]\right\}^{1 / 2}$

where $\operatorname{sign}(\mathrm{x})=1$ if $\mathrm{x}>0, \operatorname{sign}(\mathrm{x})=-1$ if $\mathrm{x}<0, \mathrm{y}_{l}$ denotes the proportion $\mathrm{n}_{l l} / \mathrm{n}_{l+}$ and $\hat{y}_{l}=\hat{r}(l)$ where $\hat{r}(l)$ is the value of the retention probability obtained from the estimated retention curve. For model deviance estimation, only class lengths containing five or more fish in total (cover + codend) were considered.

\section{Combined hauls analysis}

According to Pavez (1988) and Fryer (1991), variation between hauls using the same net could be due to a large number of uncontrolled variables, been not possible to model them explicitly. Then, the idea is to combine hauls with same mesh size opening incorporating the between haul variation and to find a mean selection curve. To do that, the next procedure was followed.

For each haul, let the parameters $v_{i}$ be independent and multivariate normal, with mean

$$
\mathrm{E}\left(\mathrm{v}_{\mathrm{i}}\right)=\mathrm{E}\left(\begin{array}{c}
\mathrm{v}_{\mathrm{i} 1} \\
\mathrm{v}_{\mathrm{i} 2}
\end{array}\right)=\left(\begin{array}{l}
\alpha_{1} \\
\alpha_{2}
\end{array}\right)
$$

and variance matrix D. Let

$$
\alpha=\left(\begin{array}{l}
\alpha_{1} \\
\alpha_{2}
\end{array}\right)
$$

then

$$
\mathrm{v}_{\mathrm{i}} \sim \mathrm{N}(\alpha, \mathrm{D}), \quad \mathrm{i}=1 \ldots \mathrm{H} .
$$

Selectivity is thus specified by the parameters $\alpha$, which determine the mean selectivity curve from $H$ hauls, and the variance matrix $D$, which measures the magnitude of the between haul variation. The parameters $v_{i}$ were estimated for each selected haul in turn. Let $\hat{v}_{i}$ be the estimate of $v_{i}$ and let $R_{i}$ be the estimate of variance matrix of $\hat{v}_{i}, i=1 \ldots H$ (obtained with the SELECT model above). Then, according with this and Eq. 6,

$$
\hat{\mathrm{v}}_{\mathrm{i}} \sim \mathrm{N}\left(\alpha, \mathrm{R}_{\mathrm{i}}+\mathrm{D}\right), \quad \mathrm{i}=1 \ldots \mathrm{H} .
$$

In Eq. 7, the variance matrix D measures the between-haul variation in the selectivity parameters $\left\{v_{i}\right\}$ and the variance matrices $\left\{R_{i}\right\}$ measure the error in estimating $\left\{\hat{\mathrm{v}}_{\mathrm{i}}\right\}$ due to the binomial variation. Eq. 7 was used to estimate $\alpha$ and $D$ with the residual maximum likelihood (REML) approach. All REML estimates were obtained by using the EM algorithm, 
which is described in detail in Appendix B of Fryer (1991).

The above-described procedure was applied only for those hauls which $l_{50}$ follow an increased trend with de size of mesh opening. To do this selection, $l_{50}$ obtained in all hauls was plotted against the mesh sizes, and a linear regression was fitted. Hauls with $l_{50}$ values above or below one SD were not considered.

\section{Master Curve analysis}

This procedure was carried out once the results were obtained in the combined haul analysis with the objective of obtaining Master Curves for males, females and combined sexes (males+females+ undetermined). Tokai \& Kitahara (1989) developed two procedures for determining the Master Curve. The first one was developed for the cover codend method and the second one for paired gears. The first approach was used in this study to determine the mesh selectivity curve for a specific mesh size of codend in trawlers from a fishing experiment with several mesh sizes. The mesh selectivity of mesh size $\mathrm{m}$ for fish of length $l$ is theoretically approximated by a function of $\mathrm{R}=\left(l-l_{0}\right) /\left(\mathrm{m}-\mathrm{m}_{0}\right)$ in the observed ranges of $l$ and $\mathrm{m}$. Here, $l_{0}$ and $\mathrm{m}_{0}$ are constants. To estimate these it is necessary to plot the selectivities at 50\% and $100 \%$ (or 90\%; Tokai et al., 1990; Tokai \& Kitahara, 1991) against mesh sizes. The intersection of two fitted re gression lines give the point $\mathrm{m}=\mathrm{m}_{0}$ and $l=l_{0}$. Finally, the retention probability is plotted against $R$, showing a smooth Master Curve, which was modeling applying the logistic model function.

All models described above were implemented in the MS-Excel spreadsheet. Nevertheless, in order to check the results we also utilized the CC 2000 SELECTIVITY program from ConStat web page (http://www.constat.dk) and the Splus code for fit this selection curves created (August 21, 1998) and facilitated by Dr. Russell Millar.

\section{RESULTS}

\section{Catch composition}

The total hauls were 9, 8, 7 and 8 for $100 \mathrm{~mm}, 110$ $\mathrm{mm}, 130 \mathrm{~mm}$ and $140 \mathrm{~mm}$ mesh size openings, respectively. The total number of individuals of hake sampled was 14,758 (49.6\% were male, $45.0 \%$ female and the remainders were undetermined). The total catch of non-target species was not significant, contributing with a mean of $1.4 \%(\mathrm{SD}=0.51)$ of total catch per haul. The non-target catch consisted largely of kingklips (Genypterus blacodes, $G$. maculatus), smallscale pomfret (Brama australis), grenadiers (Coelorhynchus sp.), small crustaceans (shrimps and prawns), and skates (Raja sp.).

The total length of Chilean hake in the catch composition ranged between $7 \mathrm{~cm}$ and $56 \mathrm{~cm}$ in the cover codend (control codend) of all hauls. In the case of experimental codends, the length ranges were $32-79 \mathrm{~cm}, 27-78 \mathrm{~cm}, 31-80 \mathrm{~cm}$ and $33-80 \mathrm{~cm}$ for $100,110,130$ and $140 \mathrm{~mm}$ of mesh opening, respectively (Fig. 1).

Total catch ranged from 1.77 tons to 10.91 tons for $100 \mathrm{~mm}$ mesh opening, $0.75-14.63$ tons (110 $\mathrm{mm}), 2.20-26.99$ tons $(130 \mathrm{~mm})$ and $0.99-11.76$ tons $(140 \mathrm{~mm})$. The proportion of fish that escaped from the experimental codends shown a trend of an exponential increase with the size of mesh opening. For $100 \mathrm{~mm}$ of mesh opening the proportion (in weight) that escaped from the codend was not more than 5\%, but it was around $25 \%$ for $140 \mathrm{~mm}$ of mesh opening and in some hauls up to $72 \%$ (circular graphs in Fig. 1).

\section{Parameter estimates and model selection by haul}

The parameter estimates ( $a, b$ and $\delta$ ), the retention length $\left(l_{25}, l_{50}\right.$ and $\left.l_{75}\right)$ and selection range SR for each size of mesh opening for the two size-selection models (Logistic and Richards) are shown in Tables 2 to 5 . For the Richards model, some fits shown matrix singularity problems in the parameter variance estimation and are not included. The $l_{50}$ values for both models shown a trend of an increase with the size of mesh opening, and the selectivity curves of larger mesh sizes are shifted to the right (Fig. 2). Also, the SR increased with mesh size up to the $130 \mathrm{~mm}$ mesh opening, and then decreased.

The likelihood ratio test was calculated to check the goodness of fit of the models (Millar, 1991). Following Millar \& Walsh (1992), and since the data contain several length classes with little or no catch, the deviance statistic (twice the log of the likelihood ratio between full and current models) was calculated using only those length-classes with a total catch of at least five fish. In all 32 cases with the logistic model there was no evidence of lack of 


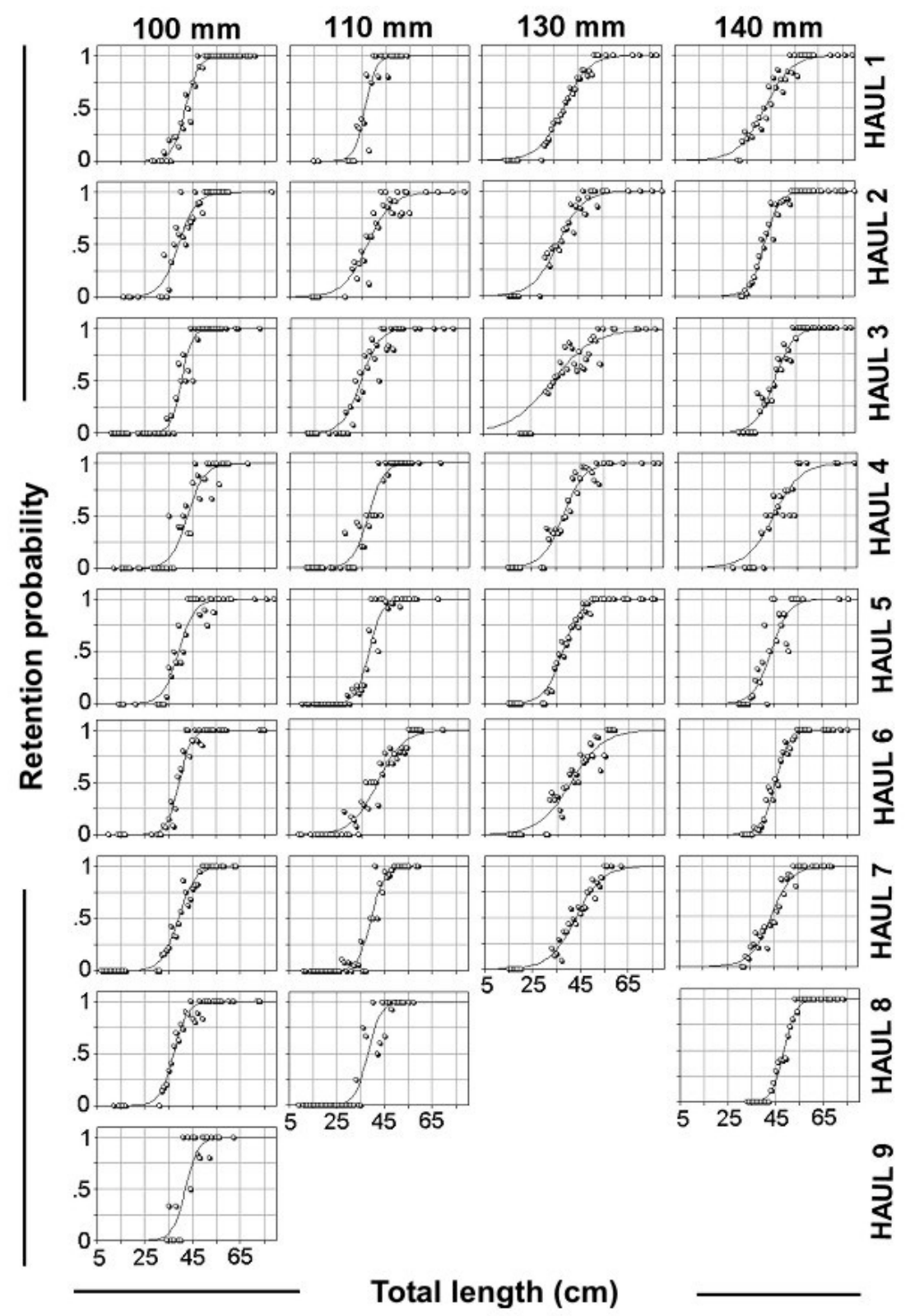

Figure 2. Proportion of catch retained (circle: observed, line: logistic model fitted) in each length class, by size of mesh opening and haul, for Chilean hake.

Figura 2. Proporción de la captura retenida (círculo: observada, línea: ajuste de modelo Logita) en cada clase de longitud de merluza común, según tamaño de luz de malla y lance. 
Table 2. Fit results with Logistic (Logit) and Richards (Rich) models by haul with $100 \mathrm{~mm}$ size of mesh opening in the codend. In parenthesis are standard errors. SR: selection range.

Tabla 2. Resultados de los ajustes por lance con el modelo Logita (Logit) y de Richards (Rich), para un tamaño de luz de malla de $100 \mathrm{~mm}$ en el copo. Los errores estándar se indican en paréntesis. SR: rango de selección.

\begin{tabular}{|c|c|c|c|c|c|c|c|c|c|c|c|c|c|c|c|c|c|c|}
\hline \multirow{2}{*}{$\begin{array}{l}\text { Haul: } \\
\text { Model: }\end{array}$} & \multicolumn{2}{|c|}{1} & \multicolumn{2}{|c|}{2} & \multirow{2}{*}{\multicolumn{2}{|c|}{$\begin{array}{c}3 \\
\text { Logit Rich }\end{array}$}} & \multirow{2}{*}{$\begin{array}{r}{ }^{4} \\
\text { Logit }\end{array}$} & \multirow[b]{2}{*}{ Rich } & \multicolumn{2}{|c|}{5} & \multicolumn{2}{|c|}{6} & \multicolumn{2}{|c|}{7} & \multicolumn{2}{|l|}{8} & \multicolumn{2}{|c|}{9} \\
\hline & Logit & Rich & Logit & Rich & & & & & Logit & Rich & Logit & Rich & Logit & Rich & Logit & Rich & Logit & Rich \\
\hline$a$ & $\begin{array}{l}-15.25 \\
(1.69)\end{array}$ & $\begin{array}{l}-30.59 \\
(13.42)\end{array}$ & $\begin{array}{l}-10.49 \\
(1.70)\end{array}$ & $\begin{array}{c}-5.65 \\
(11.47)\end{array}$ & $\begin{array}{l}-18.12 \\
(3.06)\end{array}$ & $\begin{array}{l}\ldots \\
\ldots\end{array}$ & $\begin{array}{l}-12.15 \\
(1.88)\end{array}$ & $\begin{array}{l}\cdots \\
\ldots\end{array}$ & $\begin{array}{l}-11.63 \\
(1.63)\end{array}$ & $\begin{array}{l}\cdots \\
\ldots\end{array}$ & $\begin{array}{l}-15.89 \\
(1.792\end{array}$ & $\begin{array}{l}-12.84 \\
(4.47)\end{array}$ & $\begin{array}{l}-11.11 \\
(0.88)\end{array}$ & $\begin{array}{l}-12.62 \\
(3.04)\end{array}$ & $\begin{array}{l}-12.78 \\
(1.36)\end{array}$ & $\begin{array}{l}\cdots \\
\ldots\end{array}$ & $\begin{array}{l}-15.55 \\
(3.16)\end{array}$ & $\begin{array}{l}-10.77 \\
(10.16)\end{array}$ \\
\hline$b$ & $\begin{array}{c}0.36 \\
(0.04)\end{array}$ & $\begin{array}{c}0.67 \\
(0.27)\end{array}$ & $\begin{array}{c}0.27 \\
(0.04)\end{array}$ & $\begin{array}{c}0.21 \\
(0.07)\end{array}$ & $\begin{array}{c}0.45 \\
(0.08)\end{array}$ & $\begin{array}{l}\ldots \\
\ldots\end{array}$ & $\begin{array}{c}0.28 \\
(0.04)\end{array}$ & $\begin{array}{l}\ldots \\
\ldots\end{array}$ & $\begin{array}{c}0.30 \\
(0.04)\end{array}$ & $\begin{array}{l}\ldots \\
\ldots\end{array}$ & $\begin{array}{c}0.40 \\
(0.05)\end{array}$ & $\begin{array}{c}0.35 \\
(0.08)\end{array}$ & $\begin{array}{c}0.29 \\
(0.02)\end{array}$ & $\begin{array}{c}0.31 \\
(0.06)\end{array}$ & $\begin{array}{c}0.35 \\
(0.04)\end{array}$ & $\begin{array}{l}\ldots \\
\ldots\end{array}$ & $\begin{array}{c}0.37 \\
(0.07)\end{array}$ & $\begin{array}{c}0.30 \\
(0.14)\end{array}$ \\
\hline $\mathrm{d}$ & & $\begin{array}{c}3.16 \\
(1.86)\end{array}$ & & $\begin{array}{c}0.10 \\
(0.90)\end{array}$ & & $\begin{array}{l}\ldots \\
\ldots\end{array}$ & & $\begin{array}{l}\ldots \\
\ldots\end{array}$ & & $\begin{array}{l}\ldots \\
\ldots\end{array}$ & & $\begin{array}{c}0.55 \\
(0.58)\end{array}$ & & $\begin{array}{c}1.31 \\
(0.61)\end{array}$ & & $\begin{array}{l}\cdots \\
\ldots\end{array}$ & & $\begin{array}{c}0.30 \\
(1.16)\end{array}$ \\
\hline$l_{25}(\mathrm{~cm})$ & $\begin{array}{l}38.90 \\
(0.56)\end{array}$ & $\begin{array}{l}39.35 \\
(0.68)\end{array}$ & $\begin{array}{l}34.90 \\
(1.14)\end{array}$ & $\begin{array}{l}35.10 \\
(0.88)\end{array}$ & $\begin{array}{l}37.80 \\
(0.90)\end{array}$ & $\begin{array}{l}\ldots \\
\ldots\end{array}$ & $\begin{array}{l}38.93 \\
(1.09)\end{array}$ & $\begin{array}{l}\ldots \\
\ldots\end{array}$ & $\begin{array}{l}35.35 \\
(0.80)\end{array}$ & $\begin{array}{l}\ldots \\
\ldots\end{array}$ & $\begin{array}{l}36.68 \\
(0.50)\end{array}$ & $\begin{array}{l}36.51 \\
(0.53)\end{array}$ & $\begin{array}{l}35.10 \\
(0.41)\end{array}$ & $\begin{array}{l}35.11 \\
(0.44)\end{array}$ & $\begin{array}{l}33.78 \\
(0.40)\end{array}$ & $\begin{array}{l}\ldots \\
\ldots\end{array}$ & $\begin{array}{l}39.00 \\
(1.28)\end{array}$ & $\begin{array}{l}38.65 \\
(1.41)\end{array}$ \\
\hline$l_{50}(\mathrm{~cm})$ & $\begin{array}{l}41.92 \\
(0.51)\end{array}$ & $\begin{array}{l}42.79 \\
(0.69)\end{array}$ & $\begin{array}{l}38.99 \\
(0.81)\end{array}$ & $\begin{array}{l}38.50 \\
(0.95)\end{array}$ & $\begin{array}{l}40.24 \\
(0.78)\end{array}$ & $\begin{array}{l}\ldots \\
\ldots\end{array}$ & $\begin{array}{l}42.80 \\
(0.81)\end{array}$ & $\begin{array}{l}\ldots \\
\ldots\end{array}$ & $\begin{array}{l}39.04 \\
(0.69)\end{array}$ & $\begin{array}{l}\ldots \\
\ldots\end{array}$ & $\begin{array}{l}39.40 \\
(0.50)\end{array}$ & $\begin{array}{l}39.10 \\
(0.63)\end{array}$ & $\begin{array}{l}38.95 \\
(0.36)\end{array}$ & $\begin{array}{l}39.11 \\
(0.46)\end{array}$ & $\begin{array}{l}36.96 \\
(0.28)\end{array}$ & $\begin{array}{l}\ldots \\
\ldots\end{array}$ & $\begin{array}{l}41.96 \\
(1.01)\end{array}$ & $\begin{array}{l}41.37 \\
(1.59)\end{array}$ \\
\hline$l_{75}(\mathrm{~cm})$ & $\begin{array}{l}44.94 \\
(0.65)\end{array}$ & $\begin{array}{l}45.31 \\
(0.65)\end{array}$ & $\begin{array}{l}43.07 \\
(0.91)\end{array}$ & $\begin{array}{l}42.70 \\
(1.01)\end{array}$ & $\begin{array}{l}42.68 \\
(0.87)\end{array}$ & $\begin{array}{l}\ldots \\
\ldots\end{array}$ & $\begin{array}{l}46.67 \\
(0.89)\end{array}$ & $\begin{array}{l}\ldots \\
\ldots\end{array}$ & $\begin{array}{l}42.73 \\
(0.93)\end{array}$ & $\begin{array}{l}\ldots \\
\ldots\end{array}$ & $\begin{array}{l}42.13 \\
(0.66)\end{array}$ & $\begin{array}{l}41.96 \\
(0.70)\end{array}$ & $\begin{array}{l}42.80 \\
(0.53)\end{array}$ & $\begin{array}{l}42.86 \\
(0.53)\end{array}$ & $\begin{array}{l}40.14 \\
(0.48)\end{array}$ & $\begin{array}{l}\ldots \\
\ldots\end{array}$ & $\begin{array}{l}44.93 \\
(1.05)\end{array}$ & $\begin{array}{l}44.55 \\
(1.35)\end{array}$ \\
\hline SR $(c$ & $\begin{array}{c}6.04 \\
(0.68)\end{array}$ & $\begin{array}{c}5.96 \\
(0.74)\end{array}$ & $\begin{array}{c}8.17 \\
(1.27)\end{array}$ & $\begin{array}{l}7.60 \\
(1.17)\end{array}$ & $\begin{array}{l}4.88 \\
(0.81)\end{array}$ & $\begin{array}{l}\ldots \\
\ldots\end{array}$ & $\begin{array}{c}7.74 \\
(1.16)\end{array}$ & $\begin{array}{l}\ldots \\
\ldots\end{array}$ & $\begin{array}{c}7.38 \\
(1.05)\end{array}$ & $\begin{array}{l}\ldots \\
\ldots\end{array}$ & $\begin{array}{c}5.45 \\
(0.63)\end{array}$ & $\begin{array}{c}5.45 \\
(0.62)\end{array}$ & $\begin{array}{c}7.70 \\
(0.63)\end{array}$ & $\begin{array}{c}7.75 \\
(0.64)\end{array}$ & $\begin{array}{c}6.36 \\
(0.68)\end{array}$ & $\begin{array}{l}\ldots \\
\ldots\end{array}$ & $\begin{array}{c}5.93 \\
(1.16)\end{array}$ & $\begin{array}{c}5.90 \\
(1.09)\end{array}$ \\
\hline Deviance & 23.25 & 20.60 & 13.01 & 13.32 & 6.00 & $\ldots$ & 21.47 & $\ldots$ & 19.86 & ... & 15.93 & 15.61 & 22.55 & 22.33 & 14.98 & ... & 9.68 & 8.82 \\
\hline $\begin{array}{l}\text { d.o.f. } \\
p \text {-value }\end{array}$ & $\begin{array}{c}24 \\
0.505\end{array}$ & $\begin{array}{c}23 \\
0.605\end{array}$ & $\begin{array}{c}13 \\
0.447\end{array}$ & $\begin{array}{c}12 \\
0.346\end{array}$ & $\begin{array}{c}13 \\
0.946\end{array}$ & $\begin{array}{l}\ldots \\
\ldots\end{array}$ & $\begin{array}{c}16 \\
0.161\end{array}$ & $\begin{array}{l}\ldots \\
\ldots\end{array}$ & $\begin{array}{c}14 \\
0.135\end{array}$ & $\begin{array}{l}\ldots \\
\ldots\end{array}$ & $\begin{array}{c}16 \\
0.458\end{array}$ & $\begin{array}{c}15 \\
0.408\end{array}$ & $\begin{array}{c}32 \\
0.892\end{array}$ & $\begin{array}{c}31 \\
0.872\end{array}$ & $\begin{array}{c}18 \\
0.663\end{array}$ & $\begin{array}{l}\ldots \\
\ldots\end{array}$ & $\begin{array}{c}4 \\
0.046\end{array}$ & $\begin{array}{c}3 \\
0.032\end{array}$ \\
\hline & & & & & & & & & & & & & & & & & & \\
\hline eviance & & 647 & & & ... & & .. & & & $\ldots$ & & 317 & 0.2 & 228 & $\cdots$ & & & 864 \\
\hline d.o.f. & & 1 & & & $\cdots$ & & . & & & $\ldots$ & & 1 & & & & & & 1 \\
\hline$p$-value & & 104 & & & & & r. & & & $\ldots$ & & 573 & 0.6 & 633 & $\ldots$ & & & 353 \\
\hline Hypothesis & Acce & epted & Acce & epted & $\ldots$ & & .. & . & & $\ldots$ & Acc & epted & Acce & epted & $\ldots$ & . & Acce & epted \\
\hline
\end{tabular}

(...) matrix singularity problem

Table 3. Fit results with Logistic (Logit) and Richards (Rich) models by haul with $110 \mathrm{~mm}$ size of mesh opening in the codend. In parenthesis are standard errors. SR: selection range.

Tabla 3. Resultados de los ajustes por lance con el modelo Logita (Logit) y de Richards (Rich), para un tamaño de luz de malla de $110 \mathrm{~mm}$ en el copo. Los errores estándar se indican en paréntesis. SR: rango de selección.

\begin{tabular}{|c|c|c|c|c|c|c|c|c|c|c|c|c|c|c|c|c|}
\hline \multirow{2}{*}{$\begin{array}{l}\text { Haul: } \\
\text { Model: }\end{array}$} & \multicolumn{2}{|c|}{1} & \multicolumn{2}{|c|}{2} & \multicolumn{2}{|c|}{3} & \multicolumn{2}{|c|}{4} & \multicolumn{2}{|c|}{5} & \multicolumn{2}{|c|}{6} & \multicolumn{2}{|c|}{7} & \multirow{2}{*}{\multicolumn{2}{|c|}{$\begin{array}{c}8 \\
\text { Logit Rich }\end{array}$}} \\
\hline & Logit & Rich & Logit & Rich & Logit & Rich & Logit & Rich & Logit & Rich & Logit & Rich & Logit & Rich & & \\
\hline \multirow[t]{2}{*}{$a$} & -16.08 & -15.15 & -7.37 & -4.42 & -8.96 & $\ldots$ & -11.64 & -48.44 & -14.16 & -15.01 & -7.70 & -3.38 & -14.17 & -20.30 & -14.32 & $\ldots$ \\
\hline & $(2.73)$ & $(6.84)$ & $(0.89)$ & $(5.19)$ & (1.49) & $\ldots$ & (1.87) & (38.03) & $(0.85)$ & $(2.78)$ & $(0.81)$ & $(3.75)$ & (1.31) & (6.97) & $(2.17)$ & $\ldots$ \\
\hline \multirow[t]{2}{*}{$b$} & 0.44 & 0.42 & 0.20 & 0.16 & 0.26 & $\ldots$ & 0.31 & 1.07 & 0.37 & 0.39 & 0.18 & 0.13 & 0.36 & 0.48 & 0.38 & $\ldots$ \\
\hline & $(0.07)$ & $(0.14)$ & $(0.02)$ & $(0.04)$ & $(0.04)$ & $\ldots$ & $(0.05)$ & $(0.81)$ & $(0.02)$ & $(0.06)$ & $(0.02)$ & $(0.03)$ & $(0.03)$ & $(0.14)$ & $(0.06)$ & $\ldots$ \\
\hline \multirow[t]{2}{*}{$\mathrm{d}$} & & 0.83 & & 0.25 & & $\ldots$ & & 8.16 & & 1.13 & & 0.18 & & 1.82 & & $\ldots$ \\
\hline & & (1.11) & & $(0.85)$ & & $\ldots$ & & $(7.30)$ & & $(0.40)$ & & $(0.42)$ & & $(0.35)$ & & $\ldots$ \\
\hline \multirow{2}{*}{$l_{25}(\mathrm{~cm})$} & 33.93 & 33.95 & 31.87 & 32.33 & 30.15 & $\ldots$ & 34.27 & 34.55 & 35.17 & 35.26 & 35.84 & 35.31 & 36.29 & 37.12 & 35.00 & $\ldots$ \\
\hline & $(0.73)$ & $(0.71)$ & $(1.00)$ & $(0.94)$ & (1.10) & $\ldots$ & (1.10) & (1.53) & $(0.36)$ & $(0.47)$ & $(1.00)$ & (1.02) & $(0.69)$ & $(0.99)$ & (1.17) & $\ldots$ \\
\hline \multirow[t]{2}{*}{$l_{50}(\mathrm{~cm})$} & 36.42 & 36.37 & 37.44 & 37.13 & 34.37 & $\ldots$ & 37.85 & 39.82 & 38.12 & 38.25 & 41.80 & 41.03 & 39.34 & 40.26 & 37.91 & $\ldots$ \\
\hline & $(0.52)$ & $(0.62)$ & $(0.64)$ & $(0.73)$ & $(0.70)$ & $\ldots$ & $(0.84)$ & $(0.98)$ & $(0.36)$ & $(0.54)$ & $(0.61)$ & $(0.82)$ & $(0.61)$ & (1.01) & (1.03) & $\ldots$ \\
\hline \multirow[t]{2}{*}{$l_{75}(\mathrm{~cm})$} & 38.91 & 38.88 & 43.02 & 42.83 & 38.58 & $\ldots$ & 41.42 & 42.99 & 41.08 & 41.16 & 47.77 & 47.95 & 42.39 & 42.97 & 40.82 & $\ldots$ \\
\hline & $(0.6)$ & $(0.65)$ & $(0.81)$ & $(0.88)$ & $(0.82)$ & $\ldots$ & $(0.92)$ & $(0.99)$ & $(0.45)$ & $(0.52)$ & $(0.65)$ & $(0.81)$ & $(0.65)$ & $(0.80)$ & (1.06) & $\ldots$ \\
\hline \multirow[t]{2}{*}{$\mathrm{SR}(\mathrm{cm})$} & 4.98 & 4.92 & 11.16 & 10.50 & 8.43 & $\ldots$ & 7.14 & 8.44 & 5.92 & 5.90 & 11.92 & 12.64 & 6.10 & 5.85 & 5.82 & $\ldots$ \\
\hline & $(0.83)$ & $(0.88)$ & (1.29) & (1.37) & $(1.35)$ & $\ldots$ & (1.11) & (1.67) & $(0.37)$ & $(0.37)$ & (1.17) & (1.34) & $(0.55)$ & $(0.56)$ & $(0.85)$ & $\ldots$ \\
\hline \multirow{3}{*}{$\begin{array}{c}\text { Deviance } \\
\text { d.o.f. } \\
p \text {-value }\end{array}$} & 33.10 & 33.15 & 40.17 & 39.59 & 15.94 & $\ldots$ & 9.36 & 6.99 & 38.12 & 37.99 & 27.24 & 25.03 & 14.23 & 13.24 & 7.81 & $\ldots$ \\
\hline & 19 & 18 & 22 & 21 & 17 & $\ldots$ & 14 & 13 & 32 & 31 & 23 & 22 & 29 & 28 & 19 & $\ldots$ \\
\hline & 0.023 & 0.016 & 0.010 & 0.008 & 0.528 & $\ldots$ & 0.808 & 0.903 & 0.211 & 0.181 & 0.246 & 0.296 & 0.990 & 0.992 & 0.988 & $\ldots$ \\
\hline \multicolumn{17}{|l|}{$\mathrm{H}_{0}:$ model } \\
\hline \multicolumn{3}{|l|}{ Deviance } & \multicolumn{2}{|c|}{0.587} & \multicolumn{2}{|c|}{$\ldots$} & \multicolumn{2}{|c|}{2.367} & \multicolumn{2}{|c|}{0.134} & \multicolumn{2}{|c|}{2.216} & \multicolumn{2}{|c|}{0.996} & \multicolumn{2}{|l|}{$\ldots$} \\
\hline d.o.f. & & & \multicolumn{2}{|c|}{1} & \multicolumn{2}{|c|}{$\ldots$} & \multicolumn{2}{|c|}{1} & & & 1 & & 1 & & $\ldots$ & \\
\hline$p$-value & & & 0.4 & & $\ldots$ & & 0.1 & 24 & 0.7 & 14 & 0.1 & & 0.3 & & $\ldots$ & \\
\hline Hypothesis & Acc & epted & Accel & pted & $\ldots$ & & Acce & pted & Acce & pted & Acce & pted & Acce & pted & $\ldots$ & \\
\hline
\end{tabular}

(...) matrix singularity problem 
Table 4. Fit results with Logistic (Logit) and Richards (Rich) models by haul with $130 \mathrm{~mm}$ size of mesh opening in the codend. In parenthesis are standard errors. SR: selection range.

Tabla 4. Resultados de los ajustes por lance con el modelo Logita (Logit) y de Richards (Rich), para un tamaño de luz de malla de $130 \mathrm{~mm}$ en el copo. Los errores estándar se indican en paréntesis. SR: rango de selección.

\begin{tabular}{|c|c|c|c|c|c|c|c|c|c|c|c|c|c|c|}
\hline \multirow{2}{*}{$\begin{array}{l}\text { Haul: } \\
\text { Model: }\end{array}$} & \multicolumn{2}{|c|}{1} & \multicolumn{2}{|c|}{2} & \multicolumn{2}{|c|}{3} & \multicolumn{2}{|c|}{4} & \multicolumn{2}{|c|}{5} & \multicolumn{2}{|c|}{6} & \multicolumn{2}{|c|}{7} \\
\hline & Logit & Rich & Logit & Rich & Logit & Rich & Logit & Rich & Logit & Rich & Logit & Rich & Logit & Rich \\
\hline \multirow[t]{2}{*}{$\bar{a}$} & -7.39 & -5.52 & -6.89 & -11.40 & -3.79 & $\ldots$ & -8.65 & -14.82 & -9.88 & $\ldots$ & -6.07 & -1.67 & -7.78 & -5.93 \\
\hline & $(0.64)$ & (2.99) & $(0.66)$ & $(4.20)$ & $(0.58)$ & $\ldots$ & $(0.80)$ & $(5.34)$ & $(0.83)$ & $\ldots$ & $(0.85)$ & $(9.38)$ & $(0.86)$ & $(3.83)$ \\
\hline \multirow[t]{2}{*}{$b$} & 0.19 & 0.16 & 0.19 & 0.27 & 0.11 & $\ldots$ & 0.23 & 0.34 & 0.26 & $\ldots$ & 0.15 & 0.11 & 0.18 & 0.16 \\
\hline & $(0.02)$ & $(0.04)$ & $(0.02)$ & $(0.08)$ & $(0.02)$ & $\ldots$ & $(0.02)$ & $(0.10)$ & $(0.02)$ & $\ldots$ & $(0.02)$ & $(0.03)$ & $(0.02)$ & $(0.05)$ \\
\hline \multirow[t]{2}{*}{$\delta$} & & 0.51 & & 2.59 & & $\ldots$ & & 2.71 & & $\ldots$ & & 0.08 & & 0.56 \\
\hline & & $(0.67)$ & & (1.54) & & $\ldots$ & & $(1.55)$ & & $\ldots$ & & $(0.62)$ & & $(0.81)$ \\
\hline \multirow[t]{2}{*}{$l_{25}(\mathrm{~cm})$} & 33.33 & 33.55 & 30.62 & 29.38 & 23.90 & $\ldots$ & 33.22 & 32.57 & 33.30 & $\ldots$ & 33.44 & 34.07 & 36.94 & 37.01 \\
\hline & $(0.61)$ & $(0.62)$ & $(0.71)$ & $(1.50)$ & $(1.92)$ & $\ldots$ & $(0.55)$ & $(0.96)$ & $(0.44)$ & $\ldots$ & (1.49) & $(1.31)$ & $(0.91)$ & $(0.84)$ \\
\hline \multirow{2}{*}{$l_{50}(\mathrm{~cm})$} & 39.14 & 38.95 & 36.42 & 36.67 & 33.66 & $\ldots$ & 38.05 & 38.50 & 37.46 & $\ldots$ & 40.82 & 40.44 & 43.01 & 42.81 \\
\hline & $(0.40)$ & $(0.47)$ & $(0.36)$ & $(0.43)$ & $(0.77)$ & $\ldots$ & $(0.37)$ & $(0.53)$ & $(0.35)$ & $\ldots$ & $(0.77)$ & $(0.81)$ & $(0.53)$ & $(0.66)$ \\
\hline \multirow{2}{*}{$l_{75}(\mathrm{~cm})$} & 44.96 & 44.98 & 42.23 & 42.32 & 43.43 & $\ldots$ & 42.89 & 43.05 & 41.63 & $\ldots$ & 48.21 & 48.35 & 49.08 & 49.20 \\
\hline & $(0.67)$ & $(0.71)$ & $(0.59)$ & $(0.56)$ & $(1.05)$ & $\ldots$ & $(0.62)$ & $(0.63)$ & $(0.55)$ & $\ldots$ & $(0.88)$ & (1.03) & $(0.76)$ & $(0.87)$ \\
\hline \multirow[t]{2}{*}{$\mathrm{SR}(\mathrm{cm})$} & 11.63 & 11.43 & 11.61 & 12.94 & 19.53 & $\ldots$ & 9.67 & 10.48 & 8.33 & $\ldots$ & 14.77 & 14.27 & 12.15 & 12.19 \\
\hline & (1.01) & $(1.02)$ & (1.08) & $(1.82)$ & (2.69) & $\ldots$ & $(0.90)$ & $(1.35)$ & $(0.72)$ & $\ldots$ & (1.90) & (1.83) & $(1.30)$ & $(1.30)$ \\
\hline Deviance & 7.64 & 7.55 & 30.71 & 28.14 & 30.85 & $\ldots$ & 26.39 & 22.39 & 22.41 & $\ldots$ & 21.87 & 21.54 & 14.52 & 14.39 \\
\hline d.o.f. & 20 & 19 & 21 & 20 & 21 & $\ldots$ & 21 & 20 & 18 & $\ldots$ & 18 & 17 & 20 & 19 \\
\hline$p$-value & 0.994 & 0.991 & 0.079 & 0.106 & 0.076 & $\ldots$ & 0.192 & 0.320 & 0.214 & $\ldots$ & 0.238 & 0.203 & 0.803 & 0.761 \\
\hline \multicolumn{15}{|l|}{$\mathrm{H}_{0}:$ model } \\
\hline Deviance & \multicolumn{2}{|c|}{0.085} & \multicolumn{2}{|c|}{2.563} & \multicolumn{2}{|c|}{$\ldots$} & \multicolumn{2}{|c|}{4.005} & \multicolumn{2}{|c|}{$\ldots$} & \multicolumn{2}{|c|}{0.334} & \multicolumn{2}{|c|}{0.132} \\
\hline d.o.f. & \multicolumn{2}{|c|}{1} & \multicolumn{2}{|c|}{1} & \multicolumn{2}{|c|}{$\ldots$} & \multicolumn{2}{|c|}{1} & \multicolumn{2}{|c|}{$\ldots$} & \multicolumn{2}{|c|}{1} & \multicolumn{2}{|c|}{1} \\
\hline$p$-value & 0.7 & 70 & 0.1 & 09 & .. & & 0.0 & & .. & . & 0.5 & 63 & 0.7 & \\
\hline Hypothesis & Acce & pted & Acce & pted & .. & & Acce & pted & .. & . & Acc & epted & Acce & pted \\
\hline
\end{tabular}

(...) matrix singularity problem

Table 5. Fit results with Logistic (Logit) and Richards (Rich) models by haul with $140 \mathrm{~mm}$ size of mesh opening in the codend. In parenthesis are standard errors. SR: selection range.

Tabla 5. Resultados de los ajustes por lance con el modelo Logita (Logit) y de Richards (Rich), para un tamaño de luz de malla de $140 \mathrm{~mm}$ en el copo. Los errores estándar se indican en paréntesis. SR: rango de selección.

\begin{tabular}{|c|c|c|c|c|c|c|c|c|c|c|c|c|c|c|c|c|}
\hline \multirow{2}{*}{$\begin{array}{l}\text { Haul: } \\
\text { Model: }\end{array}$} & \multicolumn{2}{|c|}{1} & \multicolumn{2}{|c|}{2} & \multicolumn{2}{|c|}{3} & \multicolumn{2}{|c|}{4} & \multicolumn{2}{|c|}{5} & \multicolumn{2}{|c|}{6} & \multicolumn{2}{|c|}{7} & \multicolumn{2}{|c|}{8} \\
\hline & Logit & Rich & Logit & Rich & Logit & Rich & Logit & Rich & Logit & Rich & Logit & Rich & Logit & Rich & Logit & Rich \\
\hline$a$ & $\begin{array}{l}-7.53 \\
(0.73)\end{array}$ & $\begin{array}{l}-8.61 \\
(4.74)\end{array}$ & $\begin{array}{l}-13.73 \\
(1.07)\end{array}$ & $\begin{array}{c}-5.16 \\
(16.20)\end{array}$ & $\begin{array}{l}-11.59 \\
(1.33)\end{array}$ & $\begin{array}{l}-11.54 \\
(4.82)\end{array}$ & $\begin{array}{l}-7.91 \\
(1.94)\end{array}$ & $\begin{array}{l}\cdots \\
\cdots\end{array}$ & $\begin{array}{l}-11.70 \\
(1.26)\end{array}$ & $\begin{array}{l}\cdots \\
\ldots\end{array}$ & $\begin{array}{l}-15.48 \\
(1.46)\end{array}$ & $\begin{array}{l}-10.88 \\
(3.94)\end{array}$ & $\begin{array}{l}-9.86 \\
(0.99)\end{array}$ & $\begin{array}{l}-25.57 \\
(12.19)\end{array}$ & $\begin{array}{l}-19.38 \\
(2.29)\end{array}$ & $\begin{array}{l}-46.95 \\
(22.92)\end{array}$ \\
\hline$b$ & $\begin{array}{c}0.18 \\
(0.02)\end{array}$ & $\begin{array}{c}0.19 \\
(0.07)\end{array}$ & $\begin{array}{c}0.32 \\
(0.03)\end{array}$ & $\begin{array}{c}0.23 \\
(0.04)\end{array}$ & $\begin{array}{c}0.25 \\
(0.03)\end{array}$ & $\begin{array}{c}0.25 \\
(0.08)\end{array}$ & $\begin{array}{c}0.17 \\
(0.04)\end{array}$ & $\begin{array}{l}\cdots \\
\cdots\end{array}$ & $\begin{array}{c}0.27 \\
(0.03)\end{array}$ & $\begin{array}{l}\cdots \\
\cdots\end{array}$ & $\begin{array}{c}0.34 \\
(0.03)\end{array}$ & $\begin{array}{c}0.27 \\
(0.06)\end{array}$ & $\begin{array}{c}0.23 \\
(0.02)\end{array}$ & $\begin{array}{c}0.51 \\
(0.23)\end{array}$ & $\begin{array}{c}0.40 \\
(0.05)\end{array}$ & $\begin{array}{c}0.90 \\
(0.43)\end{array}$ \\
\hline$\delta$ & & $\begin{array}{c}1.29 \\
(1.29)\end{array}$ & & $\begin{array}{c}0.02 \\
(0.29)\end{array}$ & & $\begin{array}{c}0.99 \\
(0.91)\end{array}$ & & $\begin{array}{l}\cdots \\
\cdots\end{array}$ & & $\begin{array}{l}\cdots \\
\cdots\end{array}$ & & $\begin{array}{c}0.39 \\
(0.44)\end{array}$ & & $\begin{array}{c}4.14 \\
(2.45)\end{array}$ & & $\begin{array}{c}4.24 \\
(2.65)\end{array}$ \\
\hline$l_{25}(\mathrm{~cm})$ & $\begin{array}{l}36.22 \\
(0.79)\end{array}$ & $\begin{array}{l}36.17 \\
(0.87)\end{array}$ & $\begin{array}{l}38.96 \\
(0.40)\end{array}$ & $\begin{array}{l}38.57 \\
(0.38)\end{array}$ & $\begin{array}{l}41.81 \\
(0.68)\end{array}$ & $\begin{array}{l}41.81 \\
(0.70)\end{array}$ & $\begin{array}{l}38.98 \\
(2.07)\end{array}$ & $\begin{array}{l}\cdots \\
\cdots\end{array}$ & $\begin{array}{l}39.57 \\
(0.62)\end{array}$ & $\begin{array}{l}\cdots \\
\cdots\end{array}$ & $\begin{array}{l}41.69 \\
(0.50)\end{array}$ & $\begin{array}{l}41.39 \\
(0.55)\end{array}$ & $\begin{array}{l}38.72 \\
(0.69)\end{array}$ & $\begin{array}{l}38.75 \\
(0.93)\end{array}$ & $\begin{array}{l}45.66 \\
(0.46)\end{array}$ & $\begin{array}{l}45.58 \\
(0.61)\end{array}$ \\
\hline$l_{50}(\mathrm{~cm})$ & $\begin{array}{l}42.41 \\
(0.59)\end{array}$ & $\begin{array}{l}42.57 \\
(0.91)\end{array}$ & $\begin{array}{l}42.35 \\
(0.36)\end{array}$ & $\begin{array}{l}41.66 \\
(0.42)\end{array}$ & $\begin{array}{l}46.19 \\
(0.43)\end{array}$ & $\begin{array}{l}46.19 \\
(0.51)\end{array}$ & $\begin{array}{l}45.27 \\
(0.97)\end{array}$ & $\begin{array}{l}\cdots \\
\cdots\end{array}$ & $\begin{array}{l}43.68 \\
(0.77)\end{array}$ & $\begin{array}{l}\cdots \\
\cdots\end{array}$ & $\begin{array}{l}44.88 \\
(0.39)\end{array}$ & $\begin{array}{l}44.49 \\
(0.52)\end{array}$ & $\begin{array}{l}43.58 \\
(0.50)\end{array}$ & $\begin{array}{l}44.46 \\
(0.58)\end{array}$ & $\begin{array}{l}48.41 \\
(0.32)\end{array}$ & $\begin{array}{l}48.91 \\
(0.37)\end{array}$ \\
\hline$l_{75}(\mathrm{~cm})$ & $\begin{array}{l}48.60 \\
(0.90)\end{array}$ & $\begin{array}{l}48.62 \\
(0.88)\end{array}$ & $\begin{array}{l}45.73 \\
(0.49)\end{array}$ & $\begin{array}{l}45.56 \\
(0.55)\end{array}$ & $\begin{array}{l}50.57 \\
(0.63)\end{array}$ & $\begin{array}{l}50.57 \\
(0.63)\end{array}$ & $\begin{array}{l}51.55 \\
(1.40)\end{array}$ & $\begin{array}{l}\cdots \\
\cdots\end{array}$ & $\begin{array}{l}47.78 \\
(1.12)\end{array}$ & $\begin{array}{l}\cdots \\
\cdots\end{array}$ & $\begin{array}{l}48.06 \\
(0.47)\end{array}$ & $\begin{array}{l}48.04 \\
(0.53)\end{array}$ & $\begin{array}{l}48.43 \\
(0.71)\end{array}$ & $\begin{array}{l}48.34 \\
(0.54)\end{array}$ & $\begin{array}{l}51.15 \\
(0.45)\end{array}$ & $\begin{array}{l}51.15 \\
(0.37)\end{array}$ \\
\hline $\mathrm{SR}(\mathrm{cm})$ & $\begin{array}{l}12.38 \\
(1.22)\end{array}$ & $\begin{array}{l}12.45 \\
(1.28)\end{array}$ & $\begin{array}{c}6.78 \\
(0.53)\end{array}$ & $\begin{array}{c}6.99 \\
(0.59)\end{array}$ & $\begin{array}{c}8.76 \\
(0.99)\end{array}$ & $\begin{array}{c}8.76 \\
(1.00)\end{array}$ & $\begin{array}{l}12.58 \\
(2.97)\end{array}$ & $\begin{array}{l}\cdots \\
\cdots\end{array}$ & $\begin{array}{c}8.20 \\
(0.96)\end{array}$ & $\begin{array}{l}\cdots \\
\cdots\end{array}$ & $\begin{array}{c}6.37 \\
(0.59)\end{array}$ & $\begin{array}{c}6.65 \\
(0.69)\end{array}$ & $\begin{array}{c}9.71 \\
(0.98)\end{array}$ & $\begin{array}{c}9.58 \\
(1.11)\end{array}$ & $\begin{array}{c}5.49 \\
(0.65)\end{array}$ & $\begin{array}{c}5.56 \\
(0.74)\end{array}$ \\
\hline Deviance & 13.85 & 15.93 & 21.36 & 15.97 & 19.06 & 19.06 & 10.47 & $\cdots$ & 20.03 & $\cdots$ & 11.56 & 10.40 & 21.78 & 17.38 & 13.60 & 9.89 \\
\hline d.o.f. & 21 & 20 & 22 & 21 & 18 & 17 & 10 & $\cdots$ & 13 & $\ldots$ & 20 & 19 & 22 & 21 & 15 & 14 \\
\hline $\begin{array}{l}p \text {-value } \\
\mathrm{H}_{0}: \text { model }\end{array}$ & 0.876 & 0.834 & 0.499 & 0.772 & 0.388 & 0.325 & 0.400 & $\ldots$ & 0.095 & $\ldots$ & 0.930 & 0.942 & 0.473 & 0.688 & 0.556 & 0.770 \\
\hline Deviance & & & & 392 & & & & & $\cdots$ & & 1.1 & 62 & 4.3 & & & 706 \\
\hline d.o.f. & & & & 1 & & & 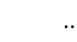 & & $\cdots$ & & 1 & 1 & & 1 & & 1 \\
\hline$p$-value & & & & 020 & & & .. & & .. & & 0.2 & 281 & 0.0 & 36 & & 054 \\
\hline Hypothesis & Acc & epted & Acc & epted & Acce & epted & .. & - & ... & & Acce & epted & Acce & pted & Acce & epted \\
\hline
\end{tabular}

(...) matrix singularity problem 
fit; the only exception was haul 2 with $110 \mathrm{~mm}$ mesh opening. With the exception of haul 2 with the 110 $\mathrm{mm}$ mesh there was also no evidence of lack of fit for the 22 cases with the Richard's model. Nonetheless, for all hypothesis tests $\left(\mathrm{H}_{0}: \delta=1\right)$ the likelihood ratio test indicated no significant differences between logistic and Richard's models, and therefore the symmetric size-selection hypothesis was not rejected (Tables 2 to 5). Therefore, only the logistic curve was considered for further analysis and the fitted curves are given in Figure 2.

\section{Mean selectivity curves}

The Figure 3 shows the linear relationship between mesh size opening and $l_{50}$, which must follow a linear increased trend. Considering this linear regression, the hauls with $l_{50}$ values above or below one SD were not considered for fitting the mean selectivity curve. The selected hauls were the numbers $1,2,3,5,6,7$ and 8 for $100 \mathrm{~mm}$ of mesh opening, the numbers 5,6 and 7 for $110 \mathrm{~mm}$, the numbers 1,6 and 7 for $130 \mathrm{~mm}$, and the numbers 1 , 2, 4, 5, 6 and 7 for $140 \mathrm{~mm}$ of mesh opening.

The estimates (with standard errors) of parameters, $l_{50}$ and $S R$ by REML are given in Table 6 and the resultant curves are shown in Figure 4, by size of mesh opening and sex. The results for Chilean hake (Fig. 4, above) showed that $l_{50}$ increased with mesh size, and also that SR tended to increase with mesh size up to $130 \mathrm{~mm}$ mesh opening. The shape of the selectivity curves for 100,110 and $140 \mathrm{~mm}$ of mesh opening were similar, and the slopes of curves were higher than the selectivity curve for 130 mm of mesh opening. The $95 \%$ confidence intervals for $l_{50}\left(\mathrm{CI}_{150}\right)$ (Fig. 4, above) with $100 \mathrm{~mm}$ and 110 $\mathrm{mm}$ of mesh opening overlap completely. The $\mathrm{CI}_{150}$ with $110 \mathrm{~mm}$ and $130 \mathrm{~mm}$ of mesh opening are partially overlapped and the $\mathrm{CI}_{150}$ with $130 \mathrm{~mm}$ and $140 \mathrm{~mm}$ of mesh opening are different.

The mean selectivity curves by sex were constructed from hauls selected previously for each mesh size. The results of these analyses are shown in Table 6 and Fig. 4. The results for males of Chilean hake (Fig. 4, middle) are very close to results obtained for both sexes, the shapes of the curves are similar, but the values of $l_{50}$ are lower (except for $140 \mathrm{~mm}$ mesh opening). Nevertheless, convergence was achieved with less than 100 iterations only in the $140 \mathrm{~mm}$ mesh opening case. Also, for females of Chilean hake (Fig. 4, below), $l_{50}$ increased with the mesh size, but the estimates were (in three over four cases) greater than for males. The shape of the $130 \mathrm{~mm}$ selectivity curve is a little different from the others, which are more «jack knife» type. The $\mathrm{CI}_{l 50}$ with $110 \mathrm{~mm}$ and $130 \mathrm{~mm}$ of mesh opening are completely overlapped. Nevertheless, these $\mathrm{CI}_{150}$ do not overlap with the $\mathrm{CI}_{150}$ for $100 \mathrm{~mm}$ and $140 \mathrm{~mm}$ of mesh opening.

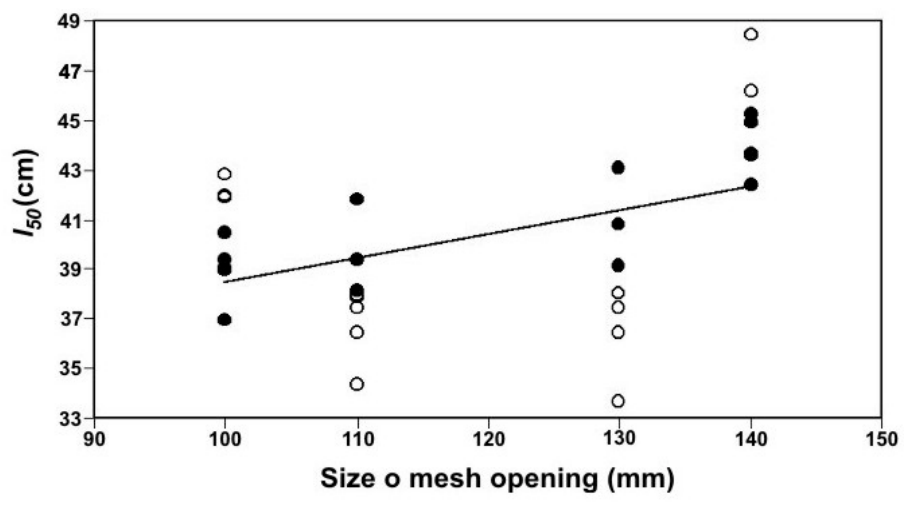

Figure 3. Size of mesh opening against $l_{50}$. The empty circles are above or below one standard deviation $(><1$ SD). $r^{2}=0.21$ and $n=32$ (full data), $r^{2}=0.68$ and $n=18$ (without the empty circle).

Figura 3. Tamaño de luz de malla versus $l_{50}$. Los círculos vacíos son superiores o inferiores a una desviación estándar ( $><1 \mathrm{SD})$. $\mathrm{r}^{2}=0,21$ y $\mathrm{n}=32$ (todos los datos), $\mathbf{r}^{2}=0,68$ y $\mathrm{n}=18$ (sin los círculos vacíos). 


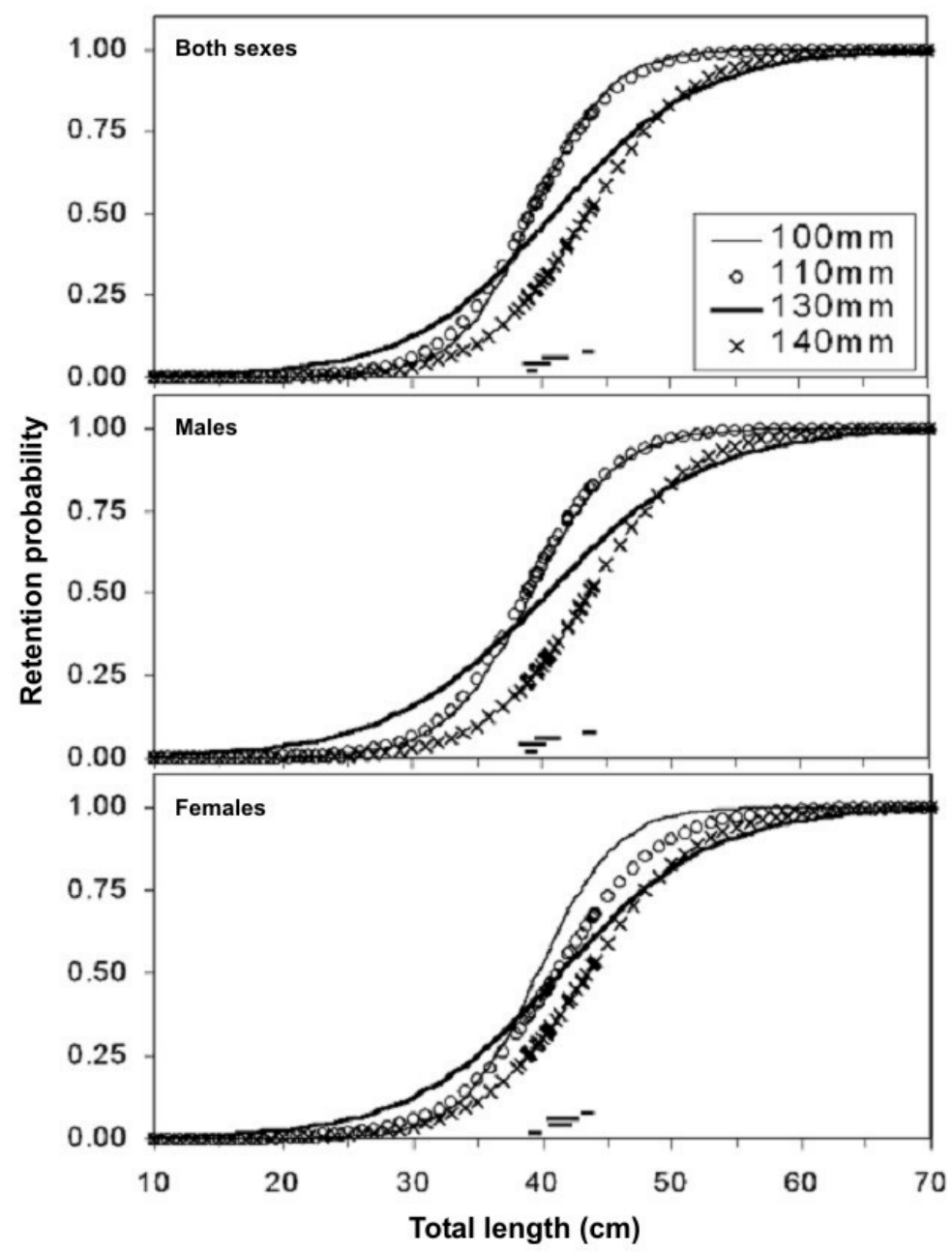

Figure 4. Chilean hake mean selectivity curves incorporating between haul variation by size of mesh opening. Going up, the thick horizontal lines indicate a $95 \%$ confidence interval for $l_{50}$ estimates of 100, 110, 130 and 140 mm mesh openings respectively.

Figura 4. Curvas de selección media de merluza común incorporando la variación entre lances, según tamaño de luz de malla. En orden ascendente, las líneas horizontales indican los intervalos de confianza al $95 \%$ de los estimados de $l_{50}$ para los tamaños de luz de malla de $100,110,130$ y 140 , respectivamente.

\section{Master Curves}

The lengths at 50 and $90 \%$ of the fitted (not observed) selectivities were plotted against mesh opening to determined $\left(\mathrm{m}_{0}, l_{0}\right)$ for total Chilean hake (3.450, 32.391), male $(2.501,30.974)$ and females (2.099, 33.317). With these values and by using the method of Tokai \& Kitahara (1989) we plot the mesh selectivities against $\mathrm{R}=\left(l-l_{0}\right) /\left(\mathrm{m}-\mathrm{m}_{0}\right)$ (Fig. 5) Applying the least squares to these plots, the respective Master Curves of mesh selectivity were estimated for total, males and females of Chilean hake as: 


$$
r(R)=r(m, l)\left\{\begin{array}{lll}
\frac{e^{\left(-2.161+2.174^{*} R\right)}}{1+e^{(-2.161+2.174 * R)}} & \text { for both sexes } & 7 \mathrm{~cm} \leq l \leq 80 \mathrm{~cm} \\
\frac{e^{\left(-2.344+2.303^{*} R\right)}}{1+e^{\left(-2.344+2.303^{*} R\right)}} & \text { for males } & 14 \mathrm{~cm} \leq l \leq 60 \mathrm{~cm} \\
\frac{e^{\left(-1.946+2.379^{*} R\right)}}{1+e^{\left(-1.946+2.379^{*} R\right)}} & \text { for females } & 13 \mathrm{~cm} \leq l \leq 80 \mathrm{~cm}
\end{array}\right.
$$
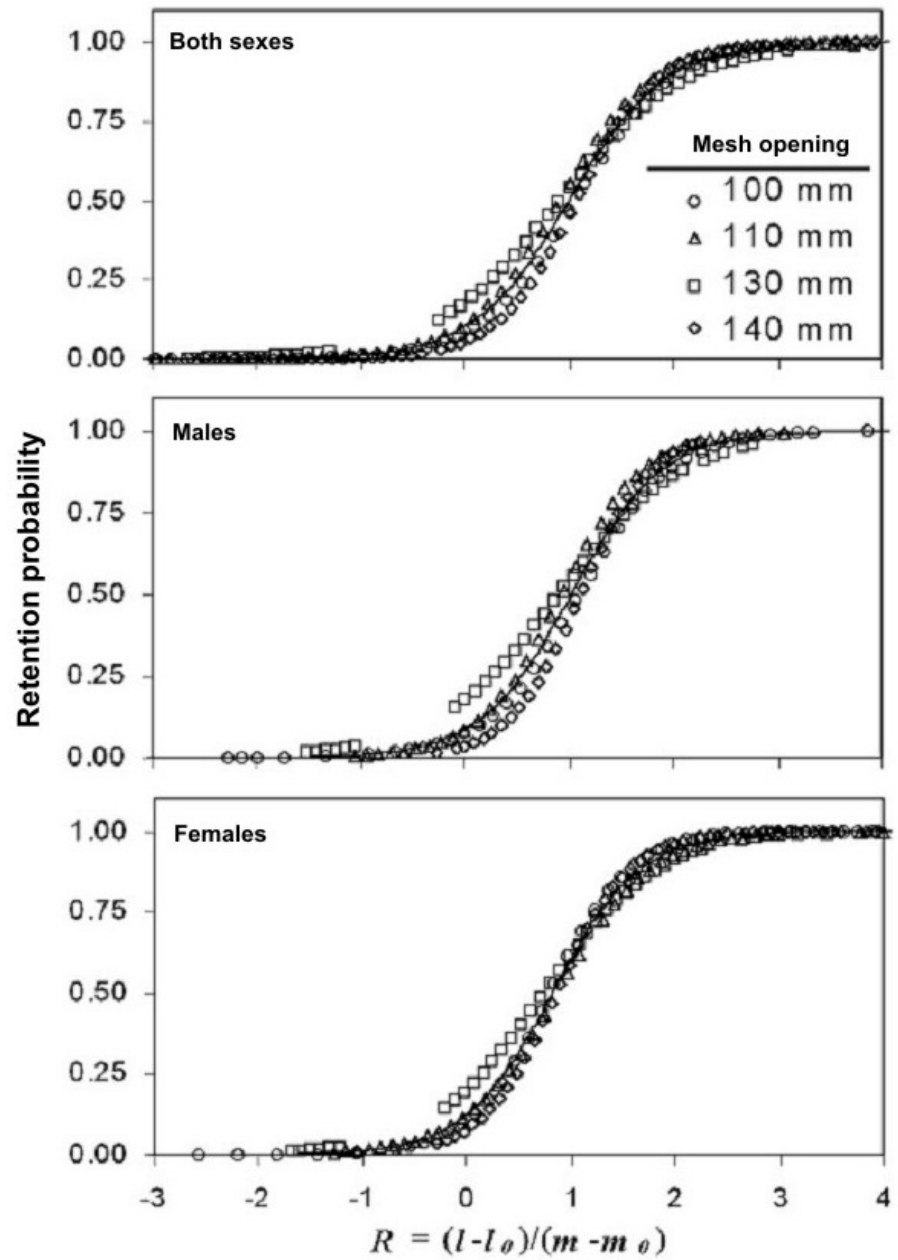

Figure 5. The mesh selectivities of $100,110,130$ and $140 \mathrm{~mm}$ mesh openings in the codend for total, male and female Chilean hake plotted against $\left(l-l_{0}\right) /\left(m-m_{0}\right)$. Solid line shows the master curves.

Figura 5. Probabilidad de retención de merluza común para los tamaños de luz de malla en el copo de 100, 110, 130 y $140 \mathrm{~mm}$ versus $\left(l-l_{0}\right) /\left(\mathbf{m}-\mathrm{m}_{0}\right)$, según sexo y total. La línea muestra la Curva Maestra. 
The Master Curves (Eq. 8) give the mesh selectivity curves of a specific mesh size between $100 \mathrm{~mm}$ and $140 \mathrm{~mm}$ of mesh opening, for total, males and females of Chilean hake. It should be noted that in Eq. 8 the size of mesh opening should be entered in centimeters.

\section{DISCUSSION}

A situation that stands out in the results is the selection curve shape for the $130 \mathrm{~mm}$ mesh opening, which is more flat than selection curves for other mesh sizes. This means that the value of the $b$ parameter is low compared with the other curves. Also, this implies that the SR for $130 \mathrm{~mm}$ mesh opening is higher than the estimated for the other mesh sizes. This is probably due to the fact that the net used in the codend construction was the only one that was not new, and the mesh size opening variation around the average (S.D.=2.9, Table 1) was high, due to the differential elongation of the twine in the mesh.

The size-selectivity of several mesh sizes can be utilized in a number of analyses. One of these is to determine the exploitation pattern at age or length, which together with data on recruitment pattern and fishing effort can give a measurement of fishing mortality rate at age or length. The fishing mortality rate vectors can be used for establishing the state of the stocks, to evaluate the effects of changes in mesh size and/or for reconstructing the age-structure of a population based on commercial catch-at-age data (Cadigan \& Millar, 1992; Wileman et al., 1996).

Another direct application is to utilize the size selectivity information in a fishing ban policy, such as MLMS regulation. Conceptually, this kind of regulation takes in mind a mixed strategy: to protect the small fish and to optimize the yield in the longterm (Jones, 1984). The first aspect implies the avoidance of the catch of juveniles, which have low contribution to the catch by weight. In the case of young fishes, his conservation means the preservation of reproductive potential of the stock, playing a fundamental role the length at $50 \%$ of maturity $\left(\mathrm{L}_{\mathrm{m}}\right)$ determination. The second aspect is the yield-per-recruit maximization, and for this the critic age $\left(\mathrm{T}_{\mathrm{mb}}\right.$, Alverson \& Carney, 1975) must be estimated. According to Pavez (1988), $\mathrm{T}_{\mathrm{mb}}$ is a disable age (or length for this purpose) for exploitation. Nevertheless, this author mentions that an operative and most realistic age is obtained considering a 80 to $90 \%$ of the $\mathrm{T}_{\mathrm{mb}}$.

If we want to apply regulation policy mentioned above with our results, it is possible to observe that big differences do not exist in the selective response of the fishing gear to males and females of hake. Nevertheless, we find a problem when $\mathrm{L}_{\mathrm{m}}$ or $\mathrm{T}_{\mathrm{mb}}$ between males and females are different. These occur because the individual growth between male and female is different, and the problem is that this obliges to determine two mesh sizes opening, one by sex. The problem is resolved when the fisheries management objective is setup clearly, and they could be: a) protect the spawning stock of females; b) protect the spawning stock of males; c) maximize the yield-per-recruit of females; d) maximize the yield-per-recruit of males; or e) some combination of these.

In the case of Chilean hake the length at $50 \%$ maturity is $37 \mathrm{~cm}$ for female (Alarcón \& Arancibia, 1993), but the size at $50 \%$ maturity for males has not yet been determined. Considering this parameter, the actual legal minimum mesh size opening (100 $\mathrm{mm})$ is adequate because $l_{50}(39.43 \mathrm{~cm}$, Table 6) is greater than $\mathrm{L}_{\mathrm{m}}$. In other words, with a $100 \mathrm{~mm}$ mesh opening it is possible protect the reproductive potential of female.

Now, we should consider the critical length. Based on age, growth and mortality estimates, Ojeda et al. (1997) give the critical age for males and females of Chilean hake, which transformed into length are $40.8 \mathrm{~cm}$ for males and $54.5 \mathrm{~cm}$ for females. With these lengths used as $l_{50}$ and utilizing the Master Curves determined here, we estimated sizes of mesh opening of $122 \mathrm{~mm}$ and $280 \mathrm{~mm}$ (extrapolated value) respectively. Obviously, if a 280 $\mathrm{mm}$ mesh opening was implemented in this fishery probably no hake would be captured (see the proportion escaping from the codend in Fig. 1). Then, it is possible to maximize the yield per recruit of males only, and for this $120 \mathrm{~mm}$ mesh opening is adequate in the codend. Also, with this mesh size the reproductive potential of the female is protected.

Unfortunately, a single regulation (minimum mesh size) does not guarantee that management objectives are reached, because several other gearconstruction factors affect the size-selectivity in the codend (Reeves et al., 1992). For instance, in order to reduce the codend diameter the fisherman put more lifting straps around the codend that reduce the mesh opening, thereby obstructing fish escapement. Large catches in short tows obstruct the 
Table 6. Mean selection curve fit results obtained by incorporating the haul variation through REML estimation by size of mesh opening and sex. Standard errors in parentheses. SR: selection range.

Tabla 6. Resultados del ajuste de la curva de selección media, por tamaño de luz de malla y sexo, obtenidos mediante la incorporación de la variación entre lances y utilizando el procedimiento REML. Los errores estándar se indican en paréntesis. SR: rango de selección.

\begin{tabular}{|c|c|c|c|c|}
\hline Opening mesh size $(\mathrm{cm})$ & 100 & 110 & 130 & 140 \\
\hline \multicolumn{5}{|l|}{ Total } \\
\hline \multirow[t]{2}{*}{$a$} & -13.430 & -11.860 & -7.226 & -10.950 \\
\hline & $(1.033)$ & $(2.106)$ & $(0.347)$ & $(1.334)$ \\
\hline \multirow[t]{2}{*}{$b$} & 0.341 & 0.302 & 0.176 & 0.251 \\
\hline & $(0.024)$ & $(0.060)$ & $(0.009)$ & $(0.030)$ \\
\hline \multirow{2}{*}{$l_{50}(\mathrm{~cm})$} & 39.35 & 39.74 & 41.14 & 43.67 \\
\hline & $(0.586)$ & $(1.123)$ & $(1.134)$ & $(0.526)$ \\
\hline \multirow[t]{2}{*}{$\mathrm{SR}(\mathrm{cm})$} & 6.42 & 7.92 & 12.20 & 9.09 \\
\hline & $(0.452)$ & $(1.903)$ & $(0.515)$ & $(1.096)$ \\
\hline \multicolumn{5}{|l|}{ Male } \\
\hline \multirow[t]{2}{*}{$a$} & -12.000 & -11.700 & -6.516 & -11.140 \\
\hline & $(0.733)$ & $(1.781)$ & $(0.533)$ & $(1.242)$ \\
\hline \multirow[t]{2}{*}{$b$} & 0.306 & 0.301 & 0.161 & 0.255 \\
\hline & $(0.019)$ & $(0.053)$ & $(0.014)$ & $(0.029)$ \\
\hline \multirow{2}{*}{$l_{50}(\mathrm{~cm})$} & 39.20 & 39.31 & 40.51 & 43.71 \\
\hline & $(0.690)$ & $(1.241)$ & $(1.050)$ & $(0.649)$ \\
\hline \multirow{2}{*}{$\mathrm{SR}(\mathrm{cm})$} & 6.70 & 7.65 & 13.35 & 8.39 \\
\hline & $(0.405)$ & $(1.497)$ & $(1.125)$ & $(0.983)$ \\
\hline \multicolumn{5}{|l|}{ Female } \\
\hline \multirow[t]{2}{*}{$a$} & -13.53 & -10.38 & -7.049 & -10.43 \\
\hline & (1.509) & (2.119) & (1.195) & (1.208) \\
\hline \multirow[t]{2}{*}{$b$} & 0.341 & 0.253 & 0.170 & 0.240 \\
\hline & $(0.036)$ & $(0.055)$ & $(0.030)$ & $(0.028)$ \\
\hline \multirow[t]{2}{*}{$l_{50}(\mathrm{~cm})$} & 39.43 & 41.34 & 41.54 & 43.43 \\
\hline & $(0.575)$ & $(0.995)$ & (1.367) & $(0.697)$ \\
\hline \multirow[t]{2}{*}{$\mathrm{SR}(\mathrm{cm})$} & 6.24 & 9.02 & 13.00 & 8.84 \\
\hline & $(0.722)$ & $(2.259)$ & $(2.458)$ & (1.018) \\
\hline
\end{tabular}

codend meshes and stretch the meshes of the extension piece of the net, also stopping fish escapement (Ehrhardt et al., 1996; Suuronen et al., 1997). Finally, the hanging ratio could be used to close the mesh stopping the hake escapes.

Despite these shortcomings, the present study has demonstrated that, obviously, an increase in mesh size produces an increase in the size selectivity and an increment in the retention lengths of Chilean hake in the bottom trawl fishery. In addition, the present study provides the selectivity Master Curve, and retention lengths can be determined for any mesh size opening between 100 and $140 \mathrm{~mm}$, being possible to evaluate other fisheries management regulations. However, an important aspect to consider is fish survival after the selection process. If all fish (or most of them) died after escape, then regulation by fixing a minimum mesh size does not achieve the objectives. If this is true, it could be important to focus on rigid size-sorting devices, because these devices could improve the Chilean hake survival and make the selectivity curve shape near to «jack knife» type, similar to occurred in other gadoid species (Gálvez, 1998, 1999).

\section{ACKNOWLEDGMENTS}

We wish to thanks Mr. Frank Stengel, Manager of Bío Bío Fishing Company, for facilities granted in conducting the field experiments and to officers and crew of the commercial fishing vessel Biomar III. Thanks are also due to Ms. Sc. Luis Cubillos for his helpful comments and suggestions. This study was supported by the project FIP N96-25 of Fisheries Research Fund - Chile. Mauricio Gálvez dedicated this work to his father's memory, Eugenio Gálvez, a selective sport fisherman, who passed away in August, 2004. 


\section{REFERENCES}

Alarcón, R. \& H. Arancibia. 1993. Talla de primera madurez sexual y fecundidad parcial en merluza común, Merluccius gayi gayi (Guichenot, 1848). Cienc. Tecnol. Mar, 16: 33-45.

Alverson, D.L. \& M.J. Carney. 1975. A graphic review of the growth and decay of population cohorts. J. Cons. Int. Explor. Mer., 36(2): 133-143.

Arana, P. 1970. Estudio sobre la selectividad de la merluza (Merluccius gayi gayi, G.) por las mallas de los artes de arrastre, en la zona de Valparaíso. Invest. Mar., 1(1): 1-39.

Cadigan, N.G. \& R.B. Millar. 1992. Reliability of selection curves obtained from trouser trawl or alternate hauls experiments. Can. J. Fish. Aquat. Sci., 49: 1624-1632.

Ehrhardt, N., R. Ercoli, J. García, J. Bartozzetti \& A. Izzo. 1996. Influencia de la cantidad de captura en la selectividad de mallas diamante y cuadrada en redes de arrastre para la merluza común (Merluccius hubbsi) e implicancias sobre el potencial de descarte. Rev. Invest. Des. Pesq., Mar del Plata, 10: 31-43.

Froese, R. \& D. Pauly (eds.). 1998. FishBase 98: concepts, design and data resources. ICLARM, Manila, Philippines, 193 pp.

Fryer, R.J. 1991. A model of between-haul variation in selectivity. ICES J. Mar. Sci., 48: 281-290.

Jones, R. 1984. Mesh size regulation and its role in fisheries management. FAO Fish. Rep., 289(Suppl. 2): 1-214.

Gálvez, M. 1998. Una alternativa de manejo: dispositivos selectivos en redes de pesca. Rev. Chile Pesq., 106: 40-45.

Gálvez, M. 1999. Método para determinar la separación entre barras y la curva de selección teórica de sistemas de grilla selectiva en redes de arrastre para peces. Invest. Mar., Valparaíso, 27: 25-38.

Klenner, A. 1978. Selectividad de copo cubierto en red de arrastre de merluza en la zona de Valparaíso. Tesis de Ingeniería Pesquera, Pontificia Universidad Católica de Valparaíso, Valparaíso, 75 pp.

Millar, R.B. 1991. Estimating the size-selectivity of fishing gears by conditioning on the total catch: the SELECT (Share Each LEngthclass's Catch Total) model. ICES. C.M. 1991/B57: 21 pp.

Millar, R.B. \& S.J. Walsh. 1992. Analysis of trawl selectivity studies with an application to trouser trawls. Fish. Res., 13: 202-220.

Ojeda, V., J. Olivares, F. Balbontín, D. Garland, L. Cubillos, R. Alarcón, M. George-Nascimento \& A. Sepúlveda. 1997. Validación de métodos aplicados en la estimación de edad y crecimiento, y determinación de la mortalidad en la merluza común en la zona centro-sur. Informe Final, FIP N ${ }^{\circ} 95-$ 15: $129 \mathrm{pp}$.

Pavez, P. 1981. Determinación del esquema de selectividad de la merluza común (Merluccius gayi gayi) por mallas de redes de arrastre de seis paneles. CORFO programa Perspectivas de Desarrollo de las Pesquerías Nacionales. Gerencia de Desarrollo (AP 82-9), Santiago, Chile.

Pavez, P. 1988. Implicaciones de una medida de regulación. Situación del tamaño mínimo de malla en redes de arrastre en la pesquería de merluza común (Merluccius gayi Guichenot, 1848). Rev. Pacífico Sur (Número Especial), pp. 717-726.

Reeves, S.A, D.W. Amstrong, R.J. Fryer \& K.A. Coull. 1992. The effects of mesh-size, cod-end extension length and cod-end diameter on the selectivity of Scottish trawls and seines. ICES J. Mar. Sci., 489: 279-288.

Saetersdal, G. \& L. Villegas. 1968. Informe sobre experimentos de selectividad de merluza con redes de arrastre. Bol. Inst. Fom. Pesq., 9: 1-16.

Subsecretaría de Pesca (SUBPESCA). 2004. Cuota global anual de captura de merluza común (Merluccius gayi gayi), año 2005. Inf. Téc. (R. Pesq.) No77, Subsecretaría de Pesca, Valparaíso. 32 pp.

Suuronen, P., D. Erickson \& E. Pikitch. 1997. Meshsize management in pelagic trawl fisheries potential solutions. In: P.A. Hancock, D.C. Smith, A. Grant \& J.P. Beumer (eds.). Proceedings of the second World Fisheries Congress, Australia, pp 563-567.

Tokai T. \& T. Kitahara. 1989. Methods of determining the mesh selectivity curve of trawlnet. Nippon Suisan Gakkaishi, 55(4): 643-649.

Tokai, T. \& T. Kitahara. 1991. Fisheries management of a small shrimp trawl in the Seto Inland Sea- 
discarded fishes and mesh size regulation. Mar. Poll. Bull., 23: 305-310.

Tokai, T., H. Ito, Y. Masaki \& T. Kitahara. 1990. Mesh selectivity curves of a shrimp beam trawl for southern rough shrimp Trachypenaeus curvirostris and mantis shrimp Oratosquilla oratoria. Nippon Suisan Gakkaishi, 56(8): 1231-1237.

Recibido: 13 enero 2005; Aceptado: 24 octubre 2005
Wileman, D.A., R.S.T. Ferro, R. Fonteyne \& R.B. Millar (eds.). 1996. Manual of methods of measuring the selectivity of towed fishing gears. ICES Coop. Res. Rep., 215: 126 pp. 